International Mathematical Forum, 1, 2006, no. 14, 665-670

\title{
Latin Squares in a Cayley Table
}

\author{
Behrooz Khosravi $^{1}$ \\ Dept. of Pure Math., Faculty of Math. and Computer Sci. \\ Amirkabir University of Technology (Tehran Polytechnic) \\ 424, Hafez Ave., Tehran 15914, Iran \\ and \\ Institute for Studies in Theoretical Physics and Mathematics (IPM) \\ khosravibbb@yahoo.com
}

\begin{abstract}
Behnam Khosravi and Bahman Khosravi
Dept. of Math., Faculty of Math. Sci.

Shahid Beheshti Univ., Evin, Tehran, 19838, Iran
\end{abstract}

\begin{abstract}
Let $G$ be a finite group of order $n \geq 2$ and $T$ be the Cayley table of $G$. Obviously $T$ is a Latin square. In this paper we study submatrices of $T$ which are Latin squares. Let $L_{m}(G)$ where $m \leq n$ be the number of Latin squares of order $m$ in $T$. We compute $L_{m}(G)$ and study some properties of such submatrices. Also we classify the Latin squares of order $m$ in $T$ and compute the number of each classes in some cases.
\end{abstract}

Mathematics Subject Classification: 20D60, 05D99

Keywords: Latin square, involution, character, Cayley table

A Latin square of order $n$ is an $n \times n$ array in which $n$ distinct symbols are arranged so that each symbol occurs once in each row and column.

Let $G$ be a finite group. Visual display of $G$ as a Cayley table is usually introduced in an elementary course on abstract algebra. Let $T$ be the Cayley table of $G$. Elementary properties of $G$ implies that $T$ be a Latin square. Also it was proved that a Latin square is a Cayley table if the composite of two rows is some row in the table $([6])$.

Let $\left(x_{1}, \ldots, x_{m}\right)$ be an $m$-tuple of elements of $G$, where $m>1$. Let $A=$ $\left(a_{i j}\right)$ be a square matrix of order $m$ such that $a_{i j}=x_{i} x_{j}$. Obviously $A$ is a

\footnotetext{
${ }^{1}$ The first author was supported in part by a grant from IPM (No. 84200024).
} 
diagonal submatrix of $T$. The investigation of how many different types of such matrices could exist and of their properties were problems solved by Freiman for $n=2,3$ (see [3]). If $K=(a, b)$ is a couple of elements of $G$ - distinct or not - then the multiplication table

$$
\left(\begin{array}{ll}
a^{2} & a b \\
b a & b^{2}
\end{array}\right)
$$

of $K$ is one of the following types

$$
\begin{aligned}
T_{0}=\left(\begin{array}{cc}
A & A \\
A & A
\end{array}\right), T_{1} & =\left(\begin{array}{ll}
A & B \\
B & A
\end{array}\right), T_{2}=\left(\begin{array}{ll}
A & B \\
B & C
\end{array}\right), \\
T_{3} & =\left(\begin{array}{ll}
A & B \\
C & A
\end{array}\right), \quad T_{4}=\left(\begin{array}{ll}
A & B \\
C & D
\end{array}\right),
\end{aligned}
$$

where equal (distinct) letters denote the same (different) elements of $K^{2}$. Denote by $P_{i}(G)=p_{i}$ the number of couples $K$ of elements of $G$ of type $T_{i}$, where $i=0,1,2,3,4$. Brailovsky and Herzog [2] have examined the case $n=2$, showing that the number $p_{i}$ of ordered couples of elements of $G$ corresponding to the matrix $A$ is a multiple of $|G|$ and determining, for $i=1,2,4$, the properties of those groups for which $p_{i}=0$. Then Bianchi and Gillio [1] extended these results and properties to the case $n \geq 3$. These results were used in determining of the bound on squares of two-sets by Slilaty and Vanderkam [7].

Since $T$ has many other submatrices it is a natural question that:

For each $m \leq n$, how many submatrices of order $m$ in $T$ are Latin squares?

In the sequel we use the following notations:

$\operatorname{Inv}(G):=$ The set of involutions of $G$,

$k:=$ The number of conjugacy classes of $G$,

$k_{i}:=$ The number of conjugacy classes of involutions in $G$,

$T:=$ The Cayley table of $G$,

$L_{m}(G):=$ The number of $m \times m$ submatrices of $T$ which are Latin squares, $L S(G):=\sum_{m|| G \mid} L_{m}(G)$.

Notation. Let $A=\left\{a_{1}, \ldots, a_{m}\right\}$ and $B=\left\{b_{1}, \ldots, b_{m}\right\}$. Let $L=\left(l_{i j}\right)$, where $l_{i j}=b_{i} a_{j},(1 \leq i, j \leq m)$, i.e.

$$
L:=\begin{array}{c|cccc} 
& a_{1} & a_{2} & \cdots & a_{m} \\
\hline b_{1} & b_{1} a_{1} & b_{1} a_{2} & \cdots & b_{1} a_{m} \\
b_{2} & b_{2} a_{1} & b_{2} a_{2} & \cdots & b_{2} a_{m} \\
\vdots & \vdots & \vdots & & \vdots \\
b_{m} & b_{m} a_{1} & b_{m} a_{2} & \cdots & b_{m} a_{m}
\end{array}
$$


Then $L$ is a $m \times m$ submatrix of $T$. Throughout this paper we denote this submatrix by $\left(b_{1}, \ldots, b_{m} ; a_{1}, \ldots, a_{m}\right)$ or simply by $(B ; A)$.

Let $r:=\#(A \cup B)$. Obviously $m \leq r \leq 2 m$. We use the notation $t_{m, r}(G)$ to denote the number of Latin squares of the form $L:=(B ; A)$ where $r:=\#(A \cup B)$. Obviously

$$
L_{m}(G)=\sum_{r=m}^{2 m} t_{m, r}(G) .
$$

The aim of this paper is to compute $L_{m}(G)$, where $m \leq n$, and study the properties of these submatrices.

Theorem 1. Let $L=\left(b_{1}, \ldots, b_{m} ; a_{1}, \ldots, a_{m}\right)$ be an $m \times m$ submatrix of the Cayley table of $G$. Then there exists a subgroup $H$ of $G$, such that $|H|=m$ and there exist $\alpha, \beta \in G$ such that $\left\{a_{1}, \ldots, a_{m}\right\}=\alpha H$ and $\left\{b_{1}, \ldots, b_{m}\right\}=\beta \alpha H \alpha^{-1}$. Also the set of elements in each row or in each column of $L$ is $\beta \alpha H$.

Proof. Let $A=\left\{a_{1}, \ldots, a_{m}\right\}, B=\left\{b_{1}, \ldots, b_{m}\right\}, \bar{A}=\left(a_{1}, \ldots, a_{m}\right)$ and $\bar{B}=$ $\left(b_{1}, \ldots, b_{m}\right)$. By the definition of $L$, the rows of $L$ are $b_{1} \bar{A}, b_{2} \bar{A}, \ldots, b_{m} \bar{A}$ and the columns of $L$ are $\bar{B} a_{1}, \bar{B} a_{2}, \ldots, \bar{B} a_{m}$. Also for every $i$ and $j$, where $1 \leq i, j \leq m$ we have $b_{i} A=B a_{j}$, since $L$ is a Latin square. Let $S:=b_{1} A$ and $H=a_{1}^{-1} A$. Now we prove that $H$ is a subgroup of $G$. Since $G$ is finite, it is enough to prove that $H$ is closed.

Let $h_{1}=a_{1}^{-1} a_{i}$ and $h_{2}=a_{1}^{-1} a_{j}$, where $1 \leq i, j \leq m$. Then $\left(a_{1}^{-1} a_{i}\right)\left(a_{1}^{-1} a_{j}\right)=$ $a_{1}^{-1}\left(a_{i} a_{1}^{-1} a_{j}\right)$. As we mentioned above, $b_{1} A=B a_{1}$. Therefore there exists some $t$ such that $b_{1} a_{i}=b_{t} a_{1}$ and so $a_{i} a_{1}^{-1}=b_{1}^{-1} b_{t}$. Since $b_{t} A=b_{1} A$, it follows that there exist some $f$ such that $1 \leq f \leq m$ and $b_{t} a_{j}=b_{1} a_{f}$. Therefore $a_{i} a_{1}^{-1} a_{j}=b_{1}^{-1} b_{t} a_{j}=b_{1}^{-1} b_{1} a_{f}=a_{f} \in A$ and so $H$ is closed. Hence $H$ is a subgroup of $G$ and $A=a_{1} H$. Since $b_{1} A=B a_{1}$, it follows that $B=b_{1} a_{1} H a_{1}^{-1}$. Therefore $S=b_{1} a_{1} H$ is the set of elements in $L$. $\diamond$

Corollary 1. If $m \nmid|G|$, then $L_{m}(G)=0$. Hence if $|G| / 2<m<|G|$, then $L_{m}(G)=0$.

Theorem 2. Let $G$ be a finite group and $p$ be a prime divisor of $|G|$. Then

$$
L_{p}(G)=\frac{t|G|^{2}}{p^{2}(p-1)},
$$

where $t$ is the number of elements of order $p$ in $G$.

Proof. By Theorem 3.1, it follows that if $L=(B ; A)$ is a Latin square of order $p$, then there exists a subgroup $H$ of order $p$ such that $A=\alpha H$ and $B=\beta \alpha H \alpha^{-1}$, where $\alpha, \beta \in G$. Therefore $A$ is a left coset of $H$ and $B$ is a right coset of $H^{\alpha}$. Hence

$$
L_{p}(G)=|G: H| \times\left|G: H^{\alpha}\right| \times s,
$$


where $s$ is the number of subgroups of order $p$. As $|H|=\left|H^{\alpha}\right|=p$, it follows that

$$
L_{p}(G)=\frac{|G|^{2}}{p^{2}} \times s .
$$

Also every $p-1$ elements of order $p$ determine a unique subgroup of order $p$ in $G$. Therefore

$$
L_{p}(G)=\frac{|G|^{2} t}{p^{2}(p-1)}
$$

where $t$ is the number of elements of order $p$ in $G$. $\diamond$

Example 1. If $S_{3}=\langle a, b\rangle$, where $a^{3}=1$ and $b^{2}=1$, then the $3 \times 3$ Latin squares are $\left(1, a, a^{2} ; 1, a, a^{2}\right),\left(1, a, a^{2} ; b, a b, a^{2} b\right),\left(b, a b, a^{2} b ; 1, a, a^{2}\right)$ and $\left(b, a b, a^{2} b ; b, a b, a^{2} b\right)$. Hence $L_{3}\left(S_{3}\right)=4$. Similarly $L_{2}\left(S_{3}\right)=27$.

If $Z_{6}=\langle x\rangle$, where $x^{6}=1$, then the $3 \times 3$ Latin submatrices are $\left(1, x^{2}, x^{4} ; 1, x^{2}, x^{4}\right),\left(x, x^{3}, x^{5} ; x, x^{3}, x^{5}\right),\left(x, x^{3}, x^{5} ; 1, x^{2}, x^{4}\right)$ and $\left(1, x^{2}, x^{4} ; x, x^{3}, x^{5}\right)$. Therefore $L_{3}\left(Z_{6}\right)=4$ but $L_{2}\left(Z_{6}\right)=9$.

Example 2. By using the above theorem it follows that $L_{2}\left(Q_{8}\right)=16$, $L_{2}\left(D_{8}\right)=80, L_{2}\left(\mathbb{Z}_{2} \times \mathbb{Z}_{2} \times \mathbb{Z}_{2}\right)=112, L_{3}\left(\mathbb{Z}_{9}\right)=9, L_{3}\left(\mathbb{Z}_{3} \times \mathbb{Z}_{3}\right)=36$.

Theorem 3. Let $G$ be a finite group. Then

(i) $L_{2}(G)=|G|^{2}|\operatorname{Inv}(G)| / 4$,

(ii) $t_{2,2}(G)=|G| k_{i} / 2$,

(iii) $t_{2,3}(G)=|G|\left(|\operatorname{Inv}(G)|-k_{i}\right) / 2$,

(iv) $t_{2,4}(G)=|G||\operatorname{Inv}(G)|(|G|-2) / 4$.

Proof. A Latin square of order 2 in the Cayley table of $G$ is of the form $(a, b ; c, d)$, where $a \neq b, c \neq d, a c=b d$ and $b c=a d$. By Theorem 1 , it follows that $\{a, b\}=\{\alpha, \alpha x\}$ and $\{c, d\}=\left\{\beta, \beta \alpha x \alpha^{-1}\right\}$, where $x$ is an involution of $G$. Also by using Theorem 2 , we have $L_{2}(G)=|G|^{2}|\operatorname{Inv}(G)| / 4$.

By the definition, $t_{2,2}(G)$ is the number of Latin squares of type $(a, b ; a, b)$ where $a$ and $b$ are different elements of $G$, i.e. $a \neq b, a b=b a$ and $a^{2}=b^{2}$. If $x=b a^{-1}$, then $x$ is an involution of $G$. Also $b=x a$ and so $a b=a x a$. As $a b=b a$, it follows that $b=a x$. Therefore $a \in C_{G}(x)$ and $b=x a$.

Conversely if $x$ is an involution and $a \in C_{G}(x)$, then a unique Latin square of type $(a, a x ; a, a x)$ is obtained and hence

$$
t_{2,2}(G)=\frac{1}{2} \sum_{x \in \operatorname{Inv}(G)}\left|C_{G}(x)\right|=k_{i}|G| / 2,
$$

where $k_{i}$ denotes the number of conjugacy classes of involutions in $G$.

Similarly $t_{2,3}(G)$ is the number of Latin squares of the form $(a, b ; a, c)$ where $a, b$ and $c$ are different elements of $G$ such that $a c=b a$ and $a^{2}=b c$. As we 
suppose that $b \neq c$, it follows that $b, c \in G \backslash C_{G}(a)$. If $x=a^{-1} b$, then $a=x c$ and $c=x a$. Hence $x \in \operatorname{Inv}(G)$ and obviously $a \notin C_{G}(a)$. Conversely let $x$ be an involution of $G$. If $a \in G \backslash C_{G}(x)$, then a Latin square of type $(a, a x ; a, x a)$ is obtained. therefore

$$
t_{2,3}(G)=\frac{1}{2} \sum_{x \in \operatorname{Inv}(G)}\left(|G|-\left|C_{G}(x)\right|\right)=\left(|\operatorname{Inv}(G)|-k_{i}\right)
$$

We know that $L_{2}(G)=\sum_{r=2}^{4} t_{2, r}(G)$, and hence

$$
t_{2,4}(G)=L_{2}(G)-t_{2,2}(G)-t_{2,3}(G)=|G||\operatorname{Inv}(G)|(|G|-2) / 4,
$$

which is the number of Latin squares of type $(a, b ; c, d)$ where $a, b, c$ and $d$ are different elements of $G$. $\diamond$

Corollary 2. Let $G$ be a finite group. Then

(i) $L_{2}(G)=0 \Leftrightarrow G$ is of odd order,

(ii) $t_{2,2}(G)=0 \Leftrightarrow G$ is of odd order,

(iii) $t_{2,3}(G)=0 \Leftrightarrow \operatorname{Inv}(G) \subset Z(G)$,

(iv) $t_{2,4}(G)=0 \Leftrightarrow$ either $G \cong \mathbb{Z}_{2}$ or $G$ is of odd order.

Proof. (i), (ii) and (iv) are immediate consequences of the above theorem. If $k_{i}=|\operatorname{Inv}(G)|$, then every element of $\operatorname{Inv}(G)$ is its conjugacy class. Hence every involution commutes with every element of $G$; i.e. every involution belongs to $Z(G)$. Therefore $\operatorname{Inv}(G) \subset Z(G)$.

Theorem 3. Let $G$ be a finite group and $m$ be a divisor of $|G|$ Then

$$
L_{m}(G)=s|G|^{2} / m^{2},
$$

where $s$ is the number of subgroups of order $m$.

Proof. Similar to the proof of Theorem 2, the result follows and we omit the proof for convenience. $\diamond$

\section{Acknowledgements}

The first author would like to thank the Institute for Studies in Theoretical Physics and Mathematics (IPM), Tehran, IRAN for the financial support.

We dedicate this paper to our parents: Professor Amir Khosravi and Mrs. Soraya Khosravi for their unending love and supports. 


\section{References}

[1] M. Bianchi and A. Gillio, Counting squares of $n$-subsets in finite groups, ARS Combinatoria 52, (1999) 97-114.

[2] L. Brailovsjy and M. Herzog, Counting squares of two-subsets in finite groups, ARS Combinatoria 42, (1996) 207-210.

[3] G. A. Freiman, On two-and three-elements subsets of groups, Aequationes Mathematicae 22, (1981) 140-152.

[4] B. Huppert, Endliche Gruppen I, Springer Verlag, Berlin (1967).

[5] I. M. Isaacs, Character theory of finite groups, Academic Press, New York, 1976.

[6] M. K. Sium Which Latin squares are Cayley tables?, Amer. Math. Monthly 98, (1991) 625-627.

[7] D. Slilaty and J. Vanderkam, Bounds on squares of two-sets, ARS Combinatoria 42, (1996) 181-191.

Received: September 11, 2005 